\title{
The recognition heuristic: a review of theory and tests
}

\section{Thorsten Pachur ${ }^{*}$, Peter M. Todd ${ }^{2}$, Gerd Gigerenzer ${ }^{3}$, Lael J. Schooler ${ }^{3}$ and Daniel G. Goldstein ${ }^{4}$}

\author{
1 Department of Psychology, University of Basel, Basel, Switzerland \\ 2 Cognitive Science Program, Indiana University, Bloomington, IN, USA \\ 3 Adaptive Behavior and Cognition, Max Planck Institute for Human Development, Berlin, Germany \\ 4 London Business School, London, UK
}

\section{Edited by:}

Norbert M. Seel, University of Freiburg, Germany

\section{Reviewed by:}

Norbert M. Seel, University of Freiburg, Germany

Andrew Howes, University of

Manchester, UK

*Correspondence:

Thorsten Pachur, Cognitive and Decision Sciences, University of Basel, Missionsstrasse 60/62, 4055 Basel,

Switzerland.

e-mail: thorsten.pachur@unibas.ch
The recognition heuristic is a prime example of how, by exploiting a match between mind and environment, a simple mental strategy can lead to efficient decision making. The proposal of the heuristic initiated a debate about the processes underlying the use of recognition in decision making. We review research addressing four key aspects of the recognition heuristic: (a) that recognition is often an ecologically valid cue; (b) that people often follow recognition when making inferences; (c) that recognition supersedes further cue knowledge; (d) that its use can produce the less-is-more effect-the phenomenon that lesser states of recognition knowledge can lead to more accurate inferences than more complete states. After we contrast the recognition heuristic to other related concepts, including availability and fluency, we carve out, from the existing findings, some boundary conditions of the use of the recognition heuristic as well as key questions for future research. Moreover, we summarize developments concerning the connection of the recognition heuristic with memory models. We suggest that the recognition heuristic is used adaptively and that, compared to other cues, recognition seems to have a special status in decision making. Finally, we discuss how systematic ignorance is exploited in other cognitive mechanisms (e.g., estimation and preference).

Keywords: heuristics, ecological rationality, memory, recognition heuristic, decision strategy

\section{INTRODUCTION}

In Sir Arthur Conan Doyle's The Final Problem, Sherlock Holmes finally faces his arch enemy, Professor Moriarty. In describing Moriarty to Watson, Holmes asks Watson, "You have probably never heard of Professor Moriarty?" "Never." "Aye, there's the genius and the wonder of the thing! The man pervades London, and no one has heard of him. That's what puts him on a pinnacle in the records of crime." Holmes thus implies that extraordinary things usually cannot avoid being heard of and thus being recognized by many people. It would be much less surprising for Watson to have never heard of Moriarty if Moriarty were insignificant. In other words, recognition may often (but not always) be useful because it can tell us something about the objects in question. Specifically, if we have heard of one object but not another, this can be an indication that the objects differ in other respects as well. Recognition would then allow us to make inferences about these other characteristics. To illustrate, imagine a culturally interested American tourist who, when planning her visit to Germany, needs to make a quick guess whether Heidelberg or Erlangen has more museums. Having heard of Heidelberg but not Erlangen, she could exploit her partial ignorance to make the (correct) inference that Heidelberg has more.

One strategy that uses recognition to make inferences from memory about the environment is what Goldstein and Gigerenzer $(1999,2002)$ called the recognition heuristic. For two-alternative choice tasks, where one has to decide which of two objects scores higher on a criterion, the heuristic can be stated as follows:

If one object is recognized, but not the other, then infer that the recognized object has a higher value on the criterion.
In situations where the recognition heuristic exploits the presence of a particular information structure - namely, that recognition knowledge about natural environments is often systematic rather than random - to make good decisions, the recognition heuristic is ecologically rational, exemplifying Herbert Simon's vision of rationality as resulting from the close fit between the mind and the environment (Simon, 1990). One condition that should govern whether this strategy will be used is therefore whether the environment is appropriately structured (meaning, as we will define later, that there is a high recognition validity). When the environment is not appropriate for using the recognition heuristic, decision makers may ignore recognition, oppose recognition, or factor in information beyond recognition, as discussed later in this article.

The exploitable relation between subjective recognition and some (not directly accessible) criterion results from a process by which the criterion influences object recognition through mediators, such as mentions in newspapers, on the Internet, on radio, on television, or by word of mouth. Specifically, objects with high criterion values tend to be mentioned more frequently in the news, frequent mentions increase the likelihood that their name will be recognized, and as a consequence, recognition becomes correlated with high criterion values (for empirical support, see Goldstein and Gigerenzer, 2002; Pachur and Hertwig, 2006; Pachur and Biele, 2007; Scheibehenne and Bröder, 2007).

Our goal in this article is to summarize and to connect research on the recognition heuristic since Goldstein and Gigerenzer (1999, 2002) first specified it, carve out from empirical tests of the recognition heuristic boundary conditions of its use, and point to novel questions that have emerged from this research. We start 
by describing and clarifying the basic characteristics and assumptions of the heuristic. For this purpose, we trace how the notion of the heuristic developed, and we locate recognition knowledge in relation to other knowledge about previous encounters with an object, such as the context of previous encounters, their frequency, and their ease of retrieval from memory (i.e., their fluency). Then we provide an overview of empirical evidence on two important issues: in what environments is the recognition heuristic ecologically rational? And do people follow recognition in these environments? We then review evidence for two bold prediction of the recognition heuristic: first, that when recognition knowledge discriminates between two objects, further cues are ignored; and second, that recognizing fewer objects can lead to higher inferential accuracy (the less-is-more effect). We close with a discussion of recent connections of the recognition heuristic with memory models and highlight relations to other judgment phenomena influenced by a previous encounter with an object.

\section{THE FOUNDATIONS AND IMPLICATIONS OF THE RECOGNITION HEURISTIC THE NON-COMPENSATORY USE OF RECOGNITION}

The recognition heuristic makes a strong claim. It assumes that if people recognize one object but not the other, and there is a substantial recognition validity, recognition is used in a non-compensatory fashion - that is, no other cues can reverse the judgment indicated by recognition (as elaborated below, the heuristic does not apply to situations in which people already have conclusive criterion knowledge about the objects, which allows a response to be deduced). To appreciate this claim, let us trace the development of the notion of the recognition heuristic. In an early article that can be considered the basis for the fast-and-frugal heuristics program, Gigerenzer et al. (1991) discussed the potential role of recognition in making bets about unknown properties of the environment. When facing a task in which one has to decide which of two objects scores higher on some criterion (e.g., which of two soccer coaches has been more successful in the past), Gigerenzer et al. (1991) proposed that people first try to solve the problem by building and using a local mental model. A local mental model can be successfully constructed if (a) precise criterion values can be retrieved from memory for both objects, (b) intervals of possible criterion values for the two objects can be retrieved that do not overlap, or (c) elementary logical operations can compensate for missing knowledge. If no such local mental model can be constructed, people construct from declarative knowledge a probabilistic mental model (PMM). Such a model consists of probabilistic cues, that is, facts about an object that are correlated with the criterion for a clearly defined set of objects. In other words, a PMM connects the specific structure of the task with the probability structure of a corresponding natural environment (stored in long-term memory) and uses the probabilistic cues to solve the problem by inductive inference. Subjective recognition of an object (which Gigerenzer et al. referred to as the "familiarity cue") was held to be one such cue.

While Gigerenzer et al. (1991) assumed that recognition functions similarly to objective cues (e.g., the cue that a city has an international airport), this view was later revised. Gigerenzer and Goldstein (1996) put forth the thesis that recognition holds a special status, because if an object is not recognized, it is not possible to retrieve cue values for that object from memory, and in this sense recognition precedes retrieval of cues. Recognition therefore serves as an initial screening step (if it correlates with the criterion, as used in the take-the-best heuristic and others) that precedes the search for further cue information. The thesis that recognition gives rise to non-compensatory processing was given prominence by Goldstein and Gigerenzer (2002), who described the recognition heuristic as follows: "the recognition heuristic is a non-compensatory strategy: if one object is recognized and the other is not, then the inference is determined; no other information can reverse the choice determined by recognition" (p. 82). "Information" here means cue values, not criterion values; in contrast, when a solution can be derived from criterion knowledge, local mental models can be applied, and the recognition heuristic does not come into play. For this reason, Goldstein and Gigerenzer (2002) did not even discuss local mental models, because their focus was on uncertain inferences as made by the recognition heuristic.

How could such a mechanism that bases a decision solely on recognition and ignores other cue knowledge make good inferences? First, recognition seems to have a retrieval primacy compared to other cue knowledge (Pachur and Hertwig, 2006). Recognition information is available to make an inference earlier than other information and enables one to make a quick and effortless decision, which is clearly beneficial when time is of the essence. Second, in some situations, information beyond recognition does not allow one to discriminate between options. For instance, customers are often unable to distinguish the taste of different beers or other products once the labels have been removed (e.g., Allison and Uhl, 1964). As a consequence, information beyond name recognition, which would take more time and effort to gather and process, may sometimes simply be useless. Third, it has been shown that the non-compensatory use of recognition can lead to more accurate inferences than mechanisms that integrate recognition with further cues (Gigerenzer and Goldstein, 1996). One reason for this is that if what is known about a recognized object is a set of negative cue values, this can lead to the object's unjustified rejection (recall that usually no knowledge can be retrieved for unrecognized objects). In a mathematical analysis of the recognition heuristic, Davis-Stober et al. (2010) identified conditions under which relying on one single cue (e.g., recognition) while ignoring further cue knowledge is actually the optimal strategy. The authors showed that as long as the cue is correlated with other cues, ignoring this further knowledge minimizes the maximal deviation from the cue weighting scheme that could be derived under perfect knowledge of the environment. Importantly, this result does not depend on the one cue being the most valid one.

Fourth, in important decision tasks during our evolutionary past, searching for information beyond recognition, even if it could be useful, may often have been dangerous. Take, for instance, foraging for food. The cost of being poisoned by sampling from unrecognized mushrooms was probably considerably higher than the cost of rejecting an unrecognized but harmless mushroom. As a consequence, an avoidance of searching for information beyond recognition could have evolved in some domains (Bullock and Todd, 1999). And some animals indeed often seem to choose food based on recognition and ignore other, potentially relevant information. For instance, Galef et al. (1990) observed that Norway rats preferred food they recognized from smelling other rats' breath over 
food they did not recognize, irrespective of whether the other rat was ill (see Noble et al., 2001, for a model of how this ignoring of further information may have evolved).

As we will discuss in greater detail below, the proposal that recognition is used in a non-compensatory fashion has led to considerable protest in the literature, with the argument that such a model would be too simple to capture human decision making. Some of this protest was due to misunderstandings of the term "noncompensatory." Although Goldstein and Gigerenzer (2002) referred in their use of the term to the judgment and decision making literature (which precisely define what it means to say that a strategy is non-compensatory), the nuances of this definition were not clear to all readers [including one of the authors of this article (Lael J. Schooler)]. Oppenheimer (2003), for instance, argued that because people seem to make judgments against recognition when they have criterion knowledge contradicting it, the recognition heuristic is not descriptive of how people make decisions. Yet the term "compensatory" refers to a trade-off between cue values, not between criterion values and cues such as recognition (Gigerenzer and Goldstein, 2011). Moreover, as mentioned before, having conclusive criterion knowledge is not a situation in which the recognition heuristic or any other inductive strategy is supposed to be used in the first place. In addition, Goldstein and Gigerenzer modeled inferences from memory in which unrecognized objects have unknown cue values. Cases in which objects are unrecognized but cue values are known (e.g., inspecting a new product in a grocery store) were not in the domain of the heuristic.

Moreover, the protest against the notion of a non-compensatory use of recognition may appear surprising, given that noncompensatory choices are commonly observed. As the authors of one classic review of 45 process studies put it, "the results firmly demonstrate that non-compensatory strategies were the dominant mode used by decision makers" (Ford et al., 1989, p. 75). Perhaps more striking is that the predictions of another memory-based heuristic, availability (Tversky and Kahneman, 1973), are also noncompensatory (based on just a single variable, e.g., speed of recall), but this seems to have bothered no one.

\section{ADAPTIVE USE OF THE RECOGNITION HEURISTIC}

Gigerenzer et al. (1999) assumed that the recognition heuristic is one of a set of strategies - the adaptive toolbox - that decision makers have at their disposal. One of the conditions in which the recognition heuristic should be applied is when recognition is (strongly) correlated with the criterion. Conversely, when recognition is only a poor cue, the recognition heuristic should not be used (at least, if a better strategy exists). To quantify the accuracy achievable by using the recognition heuristic to make criterion comparisons among a class of objects (e.g., comparing the populations of Swedish cities), Goldstein and Gigerenzer (2002) proposed the recognition validity $\alpha$. It is calculated as

$\alpha=\frac{R}{(R+W)}$,

where $R$ and $W$ equal the number of correct (right) and incorrect (wrong) inferences, respectively, that are made on all object pairs when one object is recognized and the other is not and the recognized object is judged to have the higher criterion value. The validity of object knowledge beyond recognition, which can be used to make a decision when both objects are recognized, knowledge validity $\beta$, is defined as the proportion of correct inferences among the cases where both objects are recognized.

The recognition and knowledge validities are defined relative to a reference class (Brunswik, 1943; Gigerenzer et al., 1991), which clearly specifies the population of objects that are to be judged (e.g., predicting the outcome of tennis matches at a Grand Slam tournament, or comparing the population sizes of the 50 largest British cities). To be able to make a reasonable prediction of whether people will use the recognition heuristic in a particular judgment task, it is necessary to know the reference class from which participants think the objects are drawn. Without a reference class, the recognition validity is not defined, and it is unclear how in this situation people might choose to use or suspend the heuristic. (The question of how of the recognition heuristic is selected is discussed in Low Recognition Validity and Discrediting Source Knowledge; see also Pachur and Hertwig, 2006).

\section{THE LESS-IS-MORE EFFECT}

The recognition heuristic can lead to a surprising phenomenon, in which less knowledge can lead to more accurate decisions. How is this possible? When no objects are recognized (and no other information can be gleaned from the name or image), a decision maker comparing all possible pairs of the objects can only guess which object has the greater criterion value. With an increasing number of recognized objects, there will be more and more pairs in which only one object is recognized, but also more cases in which both objects are recognized. The proportion of pairs with only one recognized object is highest when half of the objects are recognized and decreases again thereafter as a majority of objects are recognized. Now, under certain conditions, the expected accuracy of all resulting decisions (those made both with and without recognition) reaches a maximum when more than half, but fewer than all objects are recognized. When all objects are recognized, all choices have to be made based on knowledge beyond recognition, if available (because in this case the recognition heuristic is no longer applicable). Under those conditions, the accuracy of choices when all objects are recognized is lower than when at least some objects are not recognized and decision makers can benefit from the recognition heuristic's greater accuracy in this environment.

What are these conditions under which less (knowledge) can be more (accurate)? Examining the recognition heuristic analytically, Goldstein and Gigerenzer (2002) showed that a less-is-more effect will emerge in a comparison task if (but not only if) the recognition validity $(\alpha)$ is higher than the knowledge validity $(\beta)$, under the assumption that the validities are constant across different levels of the number of recognized objects, $n$ (although they showed in computer simulations that the less-is-more effect can also occur when $\alpha$ is not constant). More recently, Pachur (2010) highlighted that the less-is-more effect is strongly reduced if people who recognize more objects also have a higher knowledge validity, that is, if $n$ and the knowledge validity are positively correlated (see also Smithson, 2010). Finally, the less-is-more effect is also influenced by the quality of recognition memory. Specifically, if recognition memory is imperfect (i.e., if recognition does not always correctly 
indicate whether an object has actually been encountered or not) a less-is-more effect can occur even if the recognition validity is not higher than the knowledge validity (Katsikopoulos, 2010).

\section{INFORMATION ABOUT PREVIOUS ENCOUNTERS: WHAT RECOGNITION IS AND ISN'T}

The recognition heuristic uses information about previous encounters with an object. There are multiple dimensions of information about such encounters that can be stored (e.g., frequency, context knowledge), and even characteristics of the process of retrieving this information can be exploited for an inference (e.g., the time required to recognize an object; Schooler and Hertwig, 2005). The recognition heuristic uses only one of these various types of information: belief regarding whether or not an encounter occurred. But the term "recognition" has been applied in the literature to conceptually rather different things. Therefore, it is useful to clearly distinguish the information that the recognition heuristic employs from other forms of information about object encounters, and our intended meaning of the term recognition from other meanings.

First, "recognition" as Goldstein and Gigerenzer (2002) used it refers to the distinction "between the truly novel and the previously experienced" (p. 77). It thus differs from episodic recognition, which is commonly studied in research on recognition memory (cf. Pachur et al., 2009). In a typical recognition memory experiment, participants first study a list of items (usually existing words such as chair) and are later asked to go through a new list composed of previously studied plus unstudied items and pick out the ones that were on the original list. In other words, in these experiments typically none of the items are actually novel, because they are commonly used words. Therefore, the "mere" (or semantic) recognition that the recognition heuristic employs is insufficient to identify the correct items in this task, and knowledge about the context (i.e., episodic knowledge) in which the previously studied items were originally presented is required. The recognition heuristic does not require such episodic knowledge, because semantic recognition alone differentiates novel from previously encountered objects. Note that with novel objects, in this conception, no further cue knowledge can be available. Moreover, recognition in Goldstein and Gigerenzer's sense is not independent of a reference class. A German participant may know that she has heard of Paris, France but not Paris, Tennessee (population 10,000), and not treat Paris as recognized on a test of US cities. In addition to recognition being sensitive to a person's conception of the reference class, recognition validity, and even the decision to apply the recognition heuristic hinge on the reference class as well (see below).

A second important distinction is between (semantic) recognition and frequency information, that is, knowledge about the number of times an object has been encountered in the past (e.g., Hintzman and Curran, 1994). The recognition heuristic does not distinguish between objects one has encountered 10 times and those encountered 60 times (as long as both are recognized or unrecognized). This is one element that makes the recognition heuristic different from the availability heuristic (Tversky and Kahneman, 1973), which makes use of ease of retrieval or the quantity of recalled items (for a discussion of the different notions of availability see Hertwig et al., 2005). To make an inference, one version of the availability heuristic retrieves instances of the target event categories, such as the number of people one knows who have cancer compared to the number of people who have suffered from a stroke (Hertwig et al., 2005). The recognition heuristic, by contrast, bases an inference simply on the ability (or lack thereof) to recognize the names of the event categories.

A recognition assessment, which feeds into the recognition heuristic, unfolds over time. The speed with which this recognition assessment is made - fluency - can itself be informative and can be used to infer other facts, for instance, how frequently an object has been encountered in the past ${ }^{1}$. The recognition heuristic does not draw on fluency information and only considers whether an object is recognized or not. The notion of inferences based on recognition speed, however, has been elaborated in the fluency heuristic (Schooler and Hertwig, 2005), which uses recognition speed to distinguish between two recognized objects (i.e., where the recognition heuristic does not apply) and lends computational precision to a long tradition of research on fluency (e.g., Jacoby and Dallas, 1981; Kelley and Jacoby, 1998; Oppenheimer, 2008).

Finally, collective recognition - the proportion of people in some population who recognize an object - has been used to examine the ecological rationality of the recognition heuristic. Collective recognition has been found to be correlated with environmental quantities such as stock profitability (Borges et al., 1999; Ortmann et al., 2008) and sports success (Serwe and Frings, 2006; Pachur and Biele, 2007). Nevertheless, these tests are not direct implementations of the recognition heuristic, which models the use of individual recognition. Of course an individual could use collective recognition information (assuming he or she knows it) to make inferences about the world. However, the cognitive processes involved would be different from the recognition heuristic (e.g., including recall of the collective recognition rates or their estimation in other ways, such as by the number of people observed to have chosen some option - see Todd and Heuvelink, 2007).

To summarize, the recognition heuristic is a model of memory-based inferences. It leads to good inferences in the real world if recognition is correlated with the criterion to be inferred. The heuristic is a precisely defined algorithm that gives rise to a number of specific predictions: first, recognition determines choices even when further cues on the recognized object speak against it (i.e., non-compensatory recognition use). Second, as the recognition heuristic is assumed to be a tool in the mind's adaptive toolbox, people should apply the heuristic if recognition is substantially correlated with the criterion, but not if recognition is not predictive. And third, the recognition heuristic can produce a less-is-more effect where less knowledge can lead to higher accuracy. Next we describe empirical tests of the assumptions and predictions that the heuristic makes. In Section "Ecological Analyses of Recognition," we summarize studies that have examined the predictive power of recognition in the real world and discuss when and when not recognition is a good cue. In Section "The Recognition Heuristic as a Descriptive Model," we turn to empirical tests of people's use of the recognition heuristic.

${ }^{1}$ Fluency could thus function as a proxy for frequency information, but there is also evidence that people use both types of information independently (e.g., Schwarz and Vaughn, 2002). 
We extract from the existing studies the boundary conditions of the recognition heuristic's use, summarize evidence for the predicted non-compensatory use of recognition and the less-is-more effect.

\section{ECOLOGICAL ANALYSES OF RECOGNITION}

The recognition heuristic can be used as an ecologically rational tool from the mind's adaptive toolbox in situations where recognition is informative about a judgment to be made. Similarly, in problem solving (e.g., Simon, 1990) and in schema-based decision making (for an overview, see Goldstein and Weber, 1997) the assumption is made that recognition memory helps in quickly accessing knowledge structures that previously proved relevant in similar tasks. In what domains is recognition informative for making inferences - that is, where is it correlated with objective quantities? The degree to which recognition predicts a criterion in a given domain can be assessed in two ways. The first is to determine for a group of people their individual recognition validities $\alpha$ (based on their individual rates of recognizing the objects in a reference class) and then take the average recognition validities as an estimate of recognition's predictive value (for a critical discussion, see Katsikopoulos, 2010). A second possibility is to use the recognition responses of the group to calculate the correlation between the objects' collective recognition rates (defined as the proportion of people recognizing each object) and their criterion values, yielding the recognition correlation (Goldstein and Gigerenzer, 2002). When deviations from a perfect association between recognition rates and the criterion are due to unsystematic error (i.e., when objects with higher criterion values are as likely to be unrecognized as objects with lower criterion values are likely to be recognized), the two measures are related as follows (Pachur, 2010):

$\alpha=\frac{1+r_{\mathrm{s}}}{2}$

where $r_{\mathrm{s}}$ is the recognition correlation expressed as a Spearman rank correlation.

\section{WHEN IS RECOGNITION A GOOD PREDICTOR?}

Goldstein and Gigerenzer (2002) gave an initial overview of domains where recognition is a good predictor of particular criteria. Across a broad set of geographic domains, such as deserts, cities, lakes, and rivers, with criterion values corresponding to size or length, they found average recognition validities ranging between 0.64 and 0.95 . Since then, high recognition validities in geographic domains have been replicated repeatedly and across a number of different countries (e.g., Pohl, 2006; Pachur et al., 2008). For instance, in an analysis of the 50 largest cities of four European countries (Italy, France, England, and Spain), Pachur et al. (2008) found recognition validities between 0.72 and 0.78 .

The criterion values of objects in geographic domains (e.g., river lengths, city sizes) are relatively stable and do not change much over time (or only rather slowly), often allowing an association between people's recognition of the objects and the objects' criterion values to arise. Surprisingly, however, recognition also seems to be a valid predictor in dynamic environments. One example for a dynamic environment is sports, where new stars can rise quickly and previous ones remain well-known long after their peak performance. Serwe and
Frings (2006) assessed how well the recognition heuristic was able to forecast the winners of the tennis matches at the 2003 Wimbledon tournament. This is a difficult problem: two Association of Tennis Professionals (ATP) rankings, that consider detailed accounts of the players' past performance, predicted 66 and $68 \%$ of the matches correctly, and the seedings of the Wimbledon experts predicted $69 \%$. Serwe and Frings (2006) asked German tennis amateurs to indicate which of the tournament players they recognized. Although some of the players that the amateurs recognized were no longer very successful or were highly recognized primarily because they were also German, the recognition heuristic, using the individual recognition of players by the tennis amateurs, nonetheless correctly predicted $73 \%$ of the matches in which it could be applied and collective recognition similarly predicted $72 \%$ (for a replication see Scheibehenne and Bröder, 2007). Extensive knowledge (such as the player rankings) produced fewer correct forecasts than systematic partial ignorance (i.e., partial recognition). As knowledge in many domains is limited and partial, just like that of the amateur tennis players, the recognition heuristic can be an efficient tool for decision making when recognition is correlated with a criterion in the real world.

Further analyses have confirmed the accuracy of recognition in the sports domain. In a study on forecasts of the matches of the 2004 European Soccer Championship, Pachur and Biele (2007) asked laypeople which of the participating national teams they had heard of before. Using laypeople's recognition, the authors then found that strictly following the recognition heuristic would have led, on average, to $71 \%$ correct forecasts. However, while this was significantly better than chance performance, the authors could not replicate the finding by Serwe and Frings (2006) that recognition enables better forecasts than expert information: Fédération Internationale de Football Association (FIFA) rankings and rankings based on the previous performance of the teams achieved 85 and $89 \%$ correct forecasts, respectively. Finally, Snook and Cullen (2006) found in a study with Newfoundland students that their recognition led to an average of $85 \%$ correct judgments for the task of determining which of two National Hockey League (NHL) players had more career points.

In addition to sports, recognition has been shown to be useful in other competitive domains, such as political elections (Marewski et al., 2005), quality of US colleges (Hertwig and Todd, 2003), wealth of individual persons (Frosch et al., 2007), and performance of stocks (Borges et al., 1999; Ortmann et al., 2008; but see Boyd, 2001, for a possible restriction of that domain to rising stock markets). Thus, even in environments where objects can change their values on the criterion dimension rather quickly, recognition can prove to be a powerful predictor. Furthermore, forgetting can play a crucial role in maintaining an effective level of ignorance in such environments. To the degree that objects that objects with small criterion values are mentioned infrequently in the media and mention frequency is correlated with the activation in memory, objects with small criterion values are also more likely to be forgotten (Schooler and Hertwig, 2005).

\section{WHEN IS RECOGNITION NOT A GOOD PREDICTOR?}

Despite the apparent breadth of domains in which recognition can be exploited to infer a criterion, recognition, of course, does not predict everything. In which kinds of environments does it fail? 
First, recognition will not be correlated with criteria where people or the media talk about everything along the criterion dimension equally often (or equally rarely) or talk primarily about both ends of the dimension (e.g., very large and very small countries, or tiny and giant animals). In such cases more mentions of an object (and hence greater recognition) does not imply a high criterion value. To illustrate, Pohl (2006) found that the population of Swiss cities, but not their distance from the city Interlaken, is correlated with recognition. Correspondingly, he reported high reliance on the recognition heuristic when people were asked to judge which of two cities is larger, while when asked to judge which was closer to Interlaken, the reliance on recognition dropped to chance level.

Second (and relatedly), recognition does not seem to be a good predictor for criteria where the frequency of mentions in the media is driven by two (or more) factors that are themselves negatively correlated. Figure 1 illustrates this situation. For instance, frequent diseases are often discussed and written about because they can affect many people. At the same time, deadly or otherwise severe diseases are also often talked about - but severe diseases tend to be rather rare (Ewald, 1994). Mentions in the media and recognition of diseases are thus driven by factors that are negatively correlated (i.e., frequency of occurrence and severity). As a result, recognition is a relatively weak predictor of the frequency of occurrence of diseases: a recognized infectious disease is more common than an unrecognized one only about $60 \%$ of the time (Pachur and Hertwig, 2006). Similarly, for inferring the relative population size of animal species (a domain studied by Richter and Späth, 2006), recognition is unlikely to be a good predictor: while animal species with a large population (e.g., pigeons) are often well-known, so are endangered - and thus rare-species (e.g., pandas). Consistent with this ecological analysis, Richter and Späth (2006) reported little reliance on the recognition heuristic.

In sum, there is evidence that recognition is highly informative in many real-world domains and we are beginning to understand the conditions under which recognition is informative (though we do not have a complete theory of these conditions yet). Importantly, also other information extracted from previous encounters with objects in real-world domains seems to be informative and exploitable for making inferences, such as fluency (Hertwig et al., 2008) and

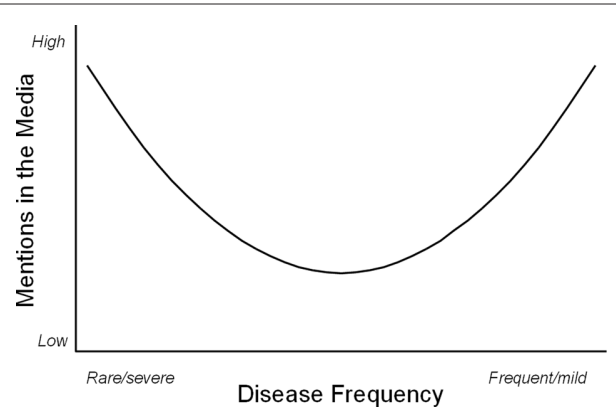

FIGURE 1 | Hypothetical plot for a task environment in which the recognition heuristic is not ecologically rational: predicting the frequency of diseases. Here, the number of mentions of a disease in the media (and thus its recognition) increases toward both extremes of the criterion dimension, for negatively correlated reasons (frequency vs. severity). As a consequence, recognition is uncorrelated with the criterion, and $\alpha$ is around 0.5 . some measures of availability (Hertwig et al., 2005). Environmental analyses are a first step in understanding the ecological rationality of decision mechanisms that use any of these sources of information.

\section{THE RECOGNITION HEURISTIC AS A DESCRIPTIVE MODEL}

Whereas the previous section reviewed findings supporting the recognition heuristic as an ecologically rational inference tool, this section provides an overview of studies that have investigated how well the model predicts human behavior. The recognition heuristic has been tested in a wide variety of domains and situations, making it possible to map more systematically the conditions under which the heuristic describes behavior more or less accurately. We will start with evidence showing that, as predicted by the recognition heuristic, many decisions align with recognition. This will be followed by a discussion of conditions under which people seem to systematically avoid basing their decisions on recognition. In the third and fourth parts of this section, we turn to tests of the recognition heuristic's bold predictions of non-compensatory processing (i.e., that all other cues beyond recognition are ignored) and that less can be more.

\section{WHEN DO PEOPLE'S DECISIONS FOLLOW RECOGNITION? The recognition heuristic in inference tasks}

In general, in domains where recognition is a good predictor (i.e., when the recognition validity $\alpha$ is high), a large proportion of people's judgments in laboratory experiments are in line with the recognition heuristic's predicted choices (typically around 90\%). Goldstein and Gigerenzer (2002) observed that when American students were asked which of two German cities is larger (a domain for which Gigerenzer and Goldstein, 1996, reported a recognition validity of 0.80 ) and they recognized one city but not the other, they picked the recognized one in $89 \%$ of the cases (and were consequently correct $71 \%$ of the time). Similarly high rates of decisions in line with recognition were found for Swiss, Belgian, Italian (Pohl, 2006), and British cities (Pachur et al., 2008), all of which are domains where the recognition validity is high. Pohl (2006; Experiment 4) found evidence for a frequent use of the recognition heuristic for other geographic materials, such as mountains, rivers, and islands.

In their application of the recognition heuristic to the sports domain, Snook and Cullen (2006) asked their participants to judge the relative number of career points achieved by different NHL players. As mentioned above, recognition is a highly useful piece of information for this task, and accordingly, a recognized player was chosen over an unrecognized one $95 \%$ of the time, even when participants had no further knowledge about the recognized player. This also led them to correct inferences $87 \%$ of the time.

\section{The recognition heuristic in forecasting tasks}

One objection to early tests of the recognition heuristic was that recognition knowledge might be confounded with criterion knowledge in inference tasks (Oppenheimer, 2003). In forecasting, by contrast, where the task is to judge a criterion that lies in the future, one cannot know the criterion for certain, making it possible to test this objection. Subsequently, it has been shown for predicting tennis matches (Serwe and Frings, 2006; Scheibehenne and Bröder, 2007), soccer games (Ayton and Önkal, 2004; Pachur and Biele, 2007), and political elections (Marewski et al., 2005) that people 
choose a recognized object over an unrecognized one even when making forecasts (around $80-90 \%$ of the time). Similarly, though not a direct test of the recognition heuristic, Weber et al. (2005) found that name recognition of a stock was associated with less perceived future riskiness, which, in turn, led to a higher tendency to decide to invest in the stock.

\section{WHEN DO PEOPLE NOT FOLLOW RECOGNITION?}

The evidence just reviewed suggests that in particular environments people might exploit the fact that they have heard of one object but not another to infer further differences between the objects. Yet an adaptive use of the recognition heuristic also requires that people do not always follow recognition. We now consider characteristics of task environments that make them inappropriate for the application of the recognition heuristic and ask whether people tend to suspend the use of the heuristic in those cases.

\section{Conclusive criterion knowledge}

As pointed out earlier, the recognition heuristic has been proposed as a mental tool for uncertain inferences (i.e., when no local mental model can be constructed). A study by Pachur and Hertwig (2006; Study 2) suggests that, indeed, people do not use recognition information when they can construct a local mental model. When asked to judge which of two infectious diseases occurs more frequently, participants systematically chose the unrecognized disease when they knew that the recognized disease was practically eradicated - in other words, when they had conclusive criterion knowledge, which allowed them to locate the recognized object at the extreme low end of the criterion dimension. To illustrate, most participants recognized leprosy and knew that leprosy is nearly eradicated. This conclusive criterion knowledge allowed them to use a local mental model to deduce that leprosy is unlikely to be the more frequent of a pair of diseases. Accordingly, when leprosy was compared with an unrecognized disease, participants judged that the unrecognized disease was more frequent in $85 \%$ of the cases.

People's ability to construct a local mental model based on conclusive criterion knowledge is also likely an explanation for the results in Oppenheimer (2003; Experiment 1). He presented Stanford students with decision tasks comparing the population sizes of nearby cities that were highly recognized but rather small (e.g., Sausalito) with fictitious cities (a diverse set of fictitious names: Al Ahbahib, Gohaiza, Heingjing, Las Besas, Papayito, Rhavadran, Rio Del Sol, Schretzburg, Svatlanov, and Weingshe). In deciding which city was larger, participants chose the recognized city in only $37 \%$ of the cases. Participants presumably knew that the nearby cities were very small (Sausalito has around 7,000 inhabitants) and inferred that the unrecognized foreign cities may be larger.

Importantly, note that the mere availability of criterion knowledge is insufficient to construct a local mental model. Rather, the criterion knowledge must be conclusive - that is, enable the decision maker to deduce a solution. For instance, knowing that Saarbrücken has a population of 190,000 (absolute criterion knowledge) or that it is the 43rd largest city in Germany (relative criterion knowledge) does not allow one to construct a local mental model and derive that Saarbrücken must be larger (or smaller) than an unrecognized city (for which no criterion knowledge can be retrieved). As described above, a local mental model can only be derived if one believes that Saarbrücken is the largest (or smallest) city in Germany and thus - by definition - larger (or smaller) than any other city; or if the subjective intervals of possible criterion values for the two objects do not overlap. Only then criterion knowledge is conclusive, obviating the need for a probabilistic mental tool such as the recognition heuristic. Accordingly, Hilbig et al. (2009) found that the mere availability of absolute and relative criterion knowledge has no or only a weak influence on the use of the recognition heuristic.

\section{Unknown reference class}

Mixing real objects with fictitious ones in an experiment or using objects from an amalgam of reference classes makes it impossible to calculate the recognition validity and thus difficult to predict whether people will use the recognition heuristic or not. For instance, Pohl (2006; Experiment 2) used a mixed set consisting of the 20 largest Swiss cities and 20 well-known but small ski resort towns. Whereas recognition is usually highly correlated with city size, the recognition of ski resorts is mainly driven by factors other than the size of the city (e.g., skiing conditions), so recognition will be useful for the set of large cities, but not for the ski resorts. Consequently, decisions in this mixed set followed recognition considerably less frequently than in Pohl's Experiment 1 using a consistent set of large cities ( 75 vs. $89 \%$ ).

Similarly, people may adopt strategies based on whether they believe that they are dealing with a representative or a biased sample of items. For instance, in addition to Oppenheimer's (2003; see also McCloy et al., 2010; Experiment 1) tests of fictional cities being compared to recognized small towns near Palo Alto, other tests compared the fictional cities to places known for specific reasons, such as Nantucket (in a limerick), Chernobyl (nuclear disaster), or Timbuktu (in an expression). Since a reference class was not provided, and because it is hard to think of a natural reference class from which places like these would constitute a representative sample, participants may correctly infer that recognition is not valid in this artificial environment. In a clearly manipulated environment, such as that of trick questions, recognition validity may be undefined. Unable to assess the ecological validity of the recognition heuristic, it is only sensible for people to elect alternative strategies.

\section{Low recognition validity}

The key condition for the adaptive use of the recognition heuristic is its ecological rationality, when recognition accurately predicts the criterion in a given environment. Figure 2 shows a summary of 11 tests of the recognition heuristic in different domains. As can be seen, people seem to follow recognition considerably less in domains where the recognition validity is very low (Pachur and Hertwig, 2006; Pohl, 2006). In fact, the average proportion of choices in line with recognition was highly correlated with the average recognition validity in the respective domain, $r=0.64$ $(p=0.03)$ - indicating an adaptive use of the recognition heuristic. These results suggest that the overall recognition validity in a particular domain is an important factor for whether the heuristic is applied or not ${ }^{2}$.

${ }^{2}$ Some results, however, suggest that people only decide not to follow recognition in domains with low recognition validity when they have alternative knowledge available that has a higher validity than recognition (Pachur and Biele, 2007; Hertwig et al., 2008). 


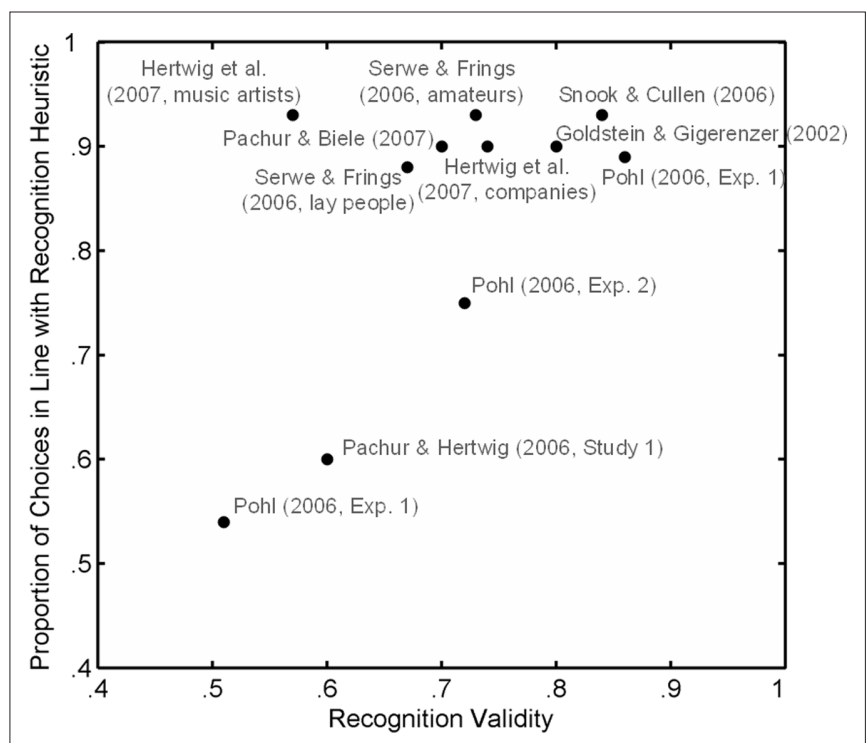

FIGURE 2 |Association between recognition validity in 11 different environments and the observed proportion of inferences following the recognition heuristic.

However, both Pohl (2006; Experiments 1 and 4, but see Experiment 2) and Pachur and Hertwig (2006) found that, looking across participants in the same domain, participants did not seem to match their reliance on recognition directly to their individual recognition validity for that domain. Specifically, the individual proportions of choices in line with the heuristic were not correlated with the individual $\alpha$. This interesting result along with the correlation depicted in Figure 2 suggests that people know about validity differences between environments, but not necessarily about the exact validity of their own recognition knowledge in particular environments. Supporting this conclusion, Pachur et al. (2008) found that although the mean of participants' estimates of the validity of their own recognition knowledge (to predict the size of British cities) matched the mean of their actual recognition validities perfectly ( 0.71 for both), the individual estimates and recognition validities were uncorrelated $(r=-0.03)$.

\section{Framing}

Another factor that seems to influence the use of the recognition heuristic is the way the inference problem is posed. In a set of studies, McCloy et al. (2010) and Hilbig et al. (2010b) compared the use of recognition in different framings of a judgment task (i.e., "Which object is larger?" vs. "Which object is smaller?"). As it turned out, participants chose the option predicted by the recognition heuristic less often when the task was to pick the object with the lower criterion value than when the task was to pick the one with the higher criterion value. Moreover, Hilbig et al. (2010b) found that participants required more time with the former than with the latter framing. There is some evidence that these asymmetries are mediated by differences in the perceived predictive value of recognition. In McCloy et al.'s (2010) study, participants rated it as more likely that they would recognize an object because it has a high criterion value than that they would not recognize an object because it has a small criterion value.

\section{Discrediting source knowledge}

According to Goldstein and Gigerenzer (2002), the rationale for the recognition heuristic's ecological rationality is the natural mediation process through which a distal criterion variable (e.g., the size of a city) increases the likelihood that the object is talked about, which, in turn, increases the likelihood that the object is recognized. Under these conditions, one can "exploit the structure of the information in natural environments" (Goldstein and Gigerenzer, 2002 , p. 76). When recognition is due to an experimental manipulation, that is, when people recognize an object from the experiment they are in, this natural mediation process is disrupted and people might use recognition differently, or not use it at all. The fact that source knowledge affects inferences has already been shown for other assessments of memory: when people believe that their memory has been manipulated experimentally or know that it is affected by factors that are completely unrelated to the criterion dimension, they rely considerably less on fluency (Jacoby et al., 1989; Hertwig et al., 2008) or ease of retrieval (e.g., Schwarz et al., 1991; Oppenheimer, 2004).

There is some indication that this is also the case for recognition. Specifically, decisions in accordance with recognition are considerably reduced when participants can attribute their sense of recognition to the experimental procedure (Newell and Shanks, 2004; Bröder and Eichler, 2006; see discussion by Pachur et al., 2008). Furthermore, knowledge that an object is recognized for a reason that has nothing to do with the object's criterion value may reduce the reliance on recognition. As mentioned above, Oppenheimer (2003; Experiment 2) found that only around 30\% of participants chose the recognized object when comparing cities such as Chernobyl and Timbuktu to unrecognized fictional ones. In addition to the fact that the reference class is undefined for fictional objects (see above), this finding might also be due to people's knowledge that the recognized city was known because of a nuclear disaster or a popular limerick rather than due to its size. In sum, people may consider available knowledge about the source of their recognition that indicates its validity for a given decision when they decide whether to follow the recognition heuristic or not.

Assessing the validity of recognition based on whether one has specific source knowledge might itself be done heuristically (cf. Johnson et al., 1993). Specifically, one might infer simply from one's ability to retrieve specific knowledge about the source of an object's recognition - for instance, that a city is recognized from a friend's description of a trip - that recognition is an unreliable cue in this case. Why? One indication that recognition is a potentially valid predictor is when an object is recognized after encountering it multiple times in many different contexts (e.g., hearing a name in several conversations with different people, or across various media), rather than through one particular, possibly biased source. Thus, easily thinking of one particular source could indicate unreliability, while difficulty in retrieving detailed information concerning a particular context in which an object was encountered could indicate that recognition has been produced by multiple sources and is therefore an ecologically valid cue. Relatedly, if an object has appeared in many different contexts, retrieving information about any specific context is associated with longer response times than when an object has appeared in only one particular context (known 
as the "fan effect" - Anderson, 1974). In other words, the fluency of retrieving a specific source might indicate whether recognition is based on a (single) biased source or not.

Given the evidence that people systematically employ the recognition heuristic in some classes of environments and not others, its use seems to involve (at least) two distinct processes. One is judging whether an object is recognized or not; the other is assessing whether recognition is a useful indicator in the given judgment task. A brain imaging study by Volz et al. (2006) obtained evidence for the neural basis of these two processes. When a decision could be made based on recognition, there was activation in the medial parietal cortex, attributed to contributions of recognition memory. In addition, there were independent changes in activation in the anterior frontomedial cortex (aFMC), a brain area involved in evaluating internal states, including self-referential processes and social-cognitive judgments (e.g., relating an aspect of the external world to oneself). The processes underlying this latter activation may be associated with evaluating whether recognition is a useful cue in the current judgment situation. Importantly, there is evidence that this evaluation occurs after the recognition judgment, or takes more cognitive resources. As Pachur and Hertwig (2006) showed, fast inferences are more likely to follow recognition than slow inferences (in an environment with low recognition validity). Similarly, compared to young adults older adults have a reduced ability to discriminate between cases where recognition is useful and where not, and this age difference is mediated by old adults' reduced cognitive capacity (Pachur et al., 2009).

\section{DOES RECOGNITION GIVE RISE TO NON-COMPENSATORY PROCESSING?}

We now review studies that have tested the most controversial prediction of the recognition heuristic - that recognition is used in a non-compensatory manner (i.e., that all other uncertain cues are ignored $)^{3}$. Importantly, the mere observation that people often pick a recognized object is not very diagnostic in this regard, as additional cues are often correlated with recognition and a consideration of this knowledge could thus lead to the same prediction as a noncompensatory mechanism based on recognition (e.g., Gigerenzer and Goldstein, 1996; Hilbig and Pohl, 2008). Moreover, people may deviate from choosing the recognized object in every case due to errors in applying the recognition heuristic. Several approaches have therefore been developed to obviate this problem: developing measures of additional knowledge use, manipulating additional cue knowledge experimentally, and making model comparisons (for a discussion, see Pachur, in press). We will discuss these three approaches separately and summarize the main findings of each.

\section{Measures of additional knowledge use}

Several authors have developed measures that reflect whether knowledge beyond recognition is used. Based on the signal detection theory framework, Pachur and Hertwig (2006; see also Pachur et al., 2009) proposed to use a $d^{\prime}$ index which expresses the degree

${ }^{3}$ We focus on studies that have examined inferences from memory, the context for which fast-and-frugal heuristics were originally proposed. Other experiments in which recognition "knowledge" was given to people along with other cues on a computer screen in an inferences-from-givens setup are not appropriate tests of this predicted non-compensatory use of recognition (e.g., Newell and Shanks's, 2004, study, in which participants were told that they recognized an imaginary company). to which people tend to follow recognition more when it leads to a correct inference than when it leads to an incorrect inference (for a very similar approach, see Hilbig and Pohl, 2008). Because according to the recognition heuristic no further cue knowledge is considered, $d^{\prime}$ should be zero. Usually, however, $d^{\prime}$ is found to be clearly larger than zero (for instance, Pachur et al., 2009, report values between 0.34 and 1.1), indicating that at least some people consider additional knowledge (although this might partly be conclusive criterion knowledge; Pachur and Hertwig, 2006; Pachur et al., 2009). More recently, Hilbig et al. (2010a) developed a formal measurement model (using a multinomial tree) that allows measuring the probability with which people ignore knowledge beyond recognition. Applying this model to several data sets, they found that people mostly use recognition in a non-compensatory way (between 62 and $76 \%$ of the time), but sometimes recruit strategies other than the recognition heuristic - strategies that do not strictly ignore additional knowledge.

\section{Experimental manipulation of additional cue knowledge}

The paradigm most frequently used for testing the non-compensatory use of recognition is to train participants on additional cue knowledge for some objects that he or she already recognizes prior to the experiment (e.g., typically cues that indicate that those objects have a small criterion value). If a person relies on the recognition heuristic and hence uses recognition in a non-compensatory way, this new knowledge beyond recognition will not affect the degree to which inferences follow recognition. That is, the recognized object should be chosen irrespective of whether the additional cue knowledge indicates that the object has a high or a low criterion value. But do people's choices conform to this prediction?

An experiment by Goldstein and Gigerenzer (2002) suggests that they do. The authors informed their US participants that in about $78 \%$ of cases, German cities that have a soccer team in the Premier League are larger than cities that do not. In addition, participants learned whether certain recognized cities had a soccer team. When later asked to pick the larger of two German cities, participants chose a recognized city over an unrecognized city in $92 \%$ of all cases even when they had learned that the recognized city had no soccer team and the additional cue information thus contradicted recognition.

Richter and Späth (2006), Newell and Fernandez (2006; Experiment 1), and Pachur et al. (2008) conducted experiments that are direct extensions of Goldstein and Gigerenzer's (2002) original study. Participants learned new information about objects that contradicted recognition (e.g., the additional learned cue indicated that the recognized city was small). Richter and Späth (2006; Experiment 3) asked their participants to judge the relative size of American cities in 190 pairs, replacing the soccer cue used in Goldstein and Gigerenzer's study with whether the city has an international airport. Without the contradictory airport cue, 17 of 28 participants followed the recognition heuristic with zero or one exception in the 32 relevant decisions, and 95\% (median 97\%) of the judgments across all participants followed the recognition heuristic - see Figure 3. When the airport cue contradicted recognition, still 17 of 28 participants made the inferences predicted by the recognition heuristic: 9 exclusively and 8 all but once (i.e., 31 out of 32 times). The median percentage of judgments in line with 

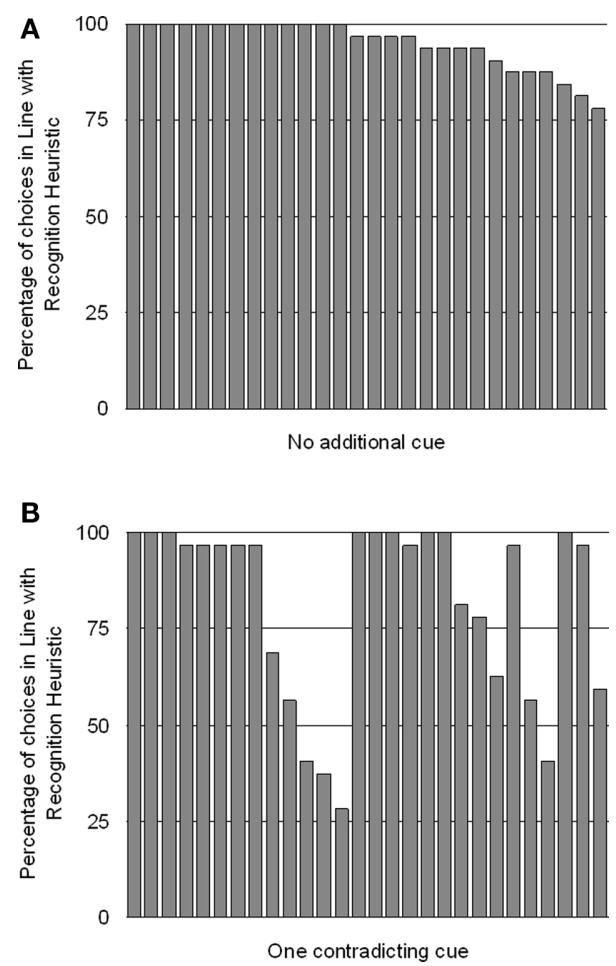

FIGURE 3 | Reanalysis of Richter and Späth's (2006) Experiment 3 based on individual data on use of recognition heuristic. The task was to infer which of two US cities has the larger population. (A) Shows the percentage of times each participant used the recognition heuristic when no contradicting cues were provided for the recognized city (with participants ordered left to right by amount of use). (B) Shows the same when participants learned that the recognized city does not have an international airport. Even when participants learned a valid cue that contradicted the recognition heuristic, a majority (17 of 28) made inferences consistent with the recognition heuristic with zero or one exceptions out of 32 decisions. (We are grateful to Richter and Späth (2006) for providing their individual data.)

the recognition heuristic remained unchanged at 97\%. The mean dropped to $82 \%$, but as Figure 3 shows, this does not mean that all individuals decreased in recognition heuristic adherence. Group means mask individual strategy selection (for similar results, see Figure 5 in Pachur et al., 2008). If we define a change as increasing or decreasing adherence by more than 1 in 32 questions, then even when facing contradictory information $43 \%$ of participants did not change, 39\% conformed to the recognition heuristic less often, and $18 \%$ conformed more often. While individual differences can be clearly seen, only 4 of 28 participants did not follow the recognition heuristic in the majority of judgments, and no participant adopted an anti-recognition strategy.

Newell and Fernandez (2006) manipulated knowledge of the probability that an unrecognized city had a soccer team (which would indicate that the city is large) and subsequently asked participants to judge the relative size of these and other, unrecognized cities. If recognition were used in a non-compensatory manner, participants' additional knowledge about whether a city has a soccer team should not affect their judgments. On the aggregate level, however, it did. The mean percentage of judgments where participants picked the recognized city was, overall, smaller when participants had learned the additional soccer team cue for that city that contradicted recognition (than when the cue supported recognition: 64 vs. $98 \%$ ), and also smaller when the probability that an unrecognized city had a soccer team was high (than when the probability was low: 77 vs. $86 \%$ ). However, as in Richter and Späth's (2006) Experiment 3, the group means mask individual differences: overall, $23 \%$ of participants always chose the recognized city, irrespective of contradicting cue information (see Pachur et al., 2008).

In the studies of Richter and Späth (2006; Experiment 2) and Pachur et al. (2008), recognition was contradicted by not just one, but by up to three cues ${ }^{4}$. Would choices still follow recognition in this situation, as predicted by the recognition heuristic? Pachur et al. (2008), whose participants were taught up to three additional cues about British cities and subsequently asked to judge the cities' relative sizes, observed higher proportions of participants ignoring further cue knowledge than using it: between 48 and $60 \%$ of their participants picked the recognized city with zero exceptions. That is, a large proportion of participants followed the choice indicated by recognition even when it was contradicted by three additional cues (for similar results, see Richter and Späth, 2006, as reanalyzed in Gigerenzer and Brighton, 2009). Importantly, this occurred although most participants perceived several of the other cues as having a higher validity than recognition.

In summary, while some analyses on the aggregate level appear to suggest that recognition is processed in a compensatory fashioninconsistent with the recognition heuristic - analyzing individual decision makers show, however, that the recognition heuristic often captures the decisions of a large proportion of people. Thus, aggregate analyses can hide important individual differences. Still, the results also indicate that some people do rely on different strategies, either compensatory or non-compensatory ones. How well does the recognition heuristic compare to alternative strategies in describing people's inferences?

\section{Model comparisons}

Whereas tests of the recognition heuristic have mainly focused on qualitative predictions of the heuristic without pitting it against other models (but see Pachur and Biele, 2007), Marewski et al. (2010) compared the recognition heuristic with several alternative models - both compensatory and non-compensatory ones. Such competitive tests are important because, as a model always has to make simplifying assumptions, its predictions will necessarily deviate from reality. Therefore, showing that data deviate from a model's prediction does not necessarily make the model irrelevant (Pachur, in press). Instead, the model remains useful as long as no other model is better able to account for the data. As it turned out from Marewski et al. (2010) comparison, none of the alternative strategies, covering a large range of different processes, was able to outperform the recognition heuristic. In other words, although people sometimes cannot help but notice cues beyond recognition, they do not seem to do this as systematically as predicted by compensatory strategies.

${ }^{4}$ The experiment by Bröder and Eichler (2006) followed a similar methodology but involved experimentally induced rather than natural recognition and so is not discussed here. 
An important insight emerging from empirical tests of the recognition heuristic is that we need to better understand individual differences in strategy use. Pachur et al. (2009) studied individual differences in how recognition is used by comparing decision making by younger and older adults, whose cognitive systems usually differ in ways potentially relevant for the use of recognition. As mentioned above, due to their reduced cognitive resources old adults have a constrained ability to judge whether recognition is useful for a given task or not - and thus are limited in their adaptive use of recognition. Such age-comparative studies on fast-and-frugal heuristics have begun to provide intriguing results concerning the adaptive use of these heuristics and their role in older adults' decision making (e.g., Mata et al., 2007).

\section{THE LESS-IS-MORE EFFECT}

Finally, what is the state of evidence for the less-is-more effect predicted (under specific conditions) by the recognition heuristic? Several demonstrations have shown that having heard of a larger number of objects is sometimes associated with lower inferential accuracy, both for individual decision makers (e.g., Goldstein and Gigerenzer, 2002) and groups (Reimer and Katsikopoulos, 2004). However, reviewing 10 data sets where the recognition validity was larger than the knowledge validity - one of the conditions for the effect highlighted by Goldstein and Gigerenzer (2002) - Pachur (2010) concluded that the evidence for the effect is mixed. Some have argued that the absence of the less-is-more effect provides evidence against people's use of the recognition heuristic (e.g., Hilbig et al., 2010a). However, Pachur (2010) also found that in many data sets, recognition and knowledge validities are correlated with the number of recognized objects, violating the condition in Goldstein and Gigerenzer's analysis that $\alpha$ and $\beta$ are constant across the number of recognized objects. Via computer simulations Pachur showed how the correlation of the recognition validity and the knowledge validity on the number of recognized objects affects the predicted existence and size of the less-is-more effect. In other words, although the recognition heuristic can lead to a less-is-more effect under certain conditions, some of these conditions seem to be rather uncommon in the real world. As a consequence, clear manifestations of the less-is-more effect may be difficult to find, even if people often use the recognition heuristic.

\section{SUMMARY}

The recognition heuristic offers a parsimonious model of how recognition is exploited in inferences from memory. Experimental tests have shown that this simple model captures several key empirical findings, such as the often dominating impact of recognition on decisions, the contingent nature of the reliance on recognition, and the counterintuitive result that limited knowledge can outwit extensive knowledge. Nevertheless, the model cannot explain every single judgment. Studies that tested the extreme hypothesis that people would rely on the recognition heuristic in 100 or $99.2 \%$ of the cases they face (e.g., Hilbig et al., 2010a) have concluded that many people violate such a pattern and appear to recruit cue knowledge beyond recognition. Such deviations from recognition heuristic use may suggest an adaptive deployment of the heuristic in some occasions that is sensitive to factors beyond the recognition validity in an environment, and a switch to other strategies that have not yet been identified. Currently we know little of the conditions under which this switch occurs and how systematically it happens; it is therefore unclear how these empirical violations of the recognition heuristic should be modeled. Also important, we need to better understand the evaluation processes preceding the heuristic's use and the reasons for the individual differences in the use of the heuristic (Pachur and Hertwig, 2006; Pachur, in press).

\section{CONNECTING THE RECOGNITION HEURISTIC TO MEMORY MODELS}

In their model of the recognition heuristic Goldstein and Gigerenzer (2002) focused on describing how information is searched for and when this search is stopped. They did not provide a model for the process underlying the recognition judgment itself. Some authors have criticized this omission (e.g., Dougherty et al., 2008). And indeed, subsequent developments have, by combining models of recognition-based inference with models of memory, given rise to new predictions of recognition-based inference that result from the processes of recognition memory. For instance, implementing the recognition heuristic within the ACT-R architecture (Anderson et al., 2004), Schooler and Hertwig (2005) have shown that the lessis-more effect can arise through forgetting. Specifically, if an object's criterion values is linked to mention frequency in the environment, which in turn influences activation in memory, small objects are more likely to decay in activation and thus become unrecognized than large objects. As a result of this systematic pattern in forgetting, memory decay can increase the recognition validity. In further ACT-R analyses, Marewski and Schooler (in press) show that the recognition heuristic is most likely to produce accurate inferences when knowledge is available about the recognized object.

A second example is Dougherty et al.'s (2008) work on the recognition heuristic using Hintzman's MINERVA model (see also Pachur, 2010). In computer simulations, the authors identified alternative accounts for the less-is-more effect and demonstrated that the effect can also occur if inferences are based on a mechanism using continuous familiarity. Third, Erdfelder et al. (2011) connected the recognition heuristic with a two-high-threshold memory model, that also accounts for the process underlying the recognition judgment. The authors showed that this combined model can explain response time patterns that Hilbig and Pohl (2009) interpreted as evidence for a compensatory use of recognition. Finally, using a signal detection theory framework several authors have analyzed the implications of specific performance patterns in recognition memory (i.e., hit and false alarm rates) for the accuracy of the recognition heuristic (Pleskac, 2007; Katsikopoulos, 2010). For instance, extending Goldstein and Gigerenzer's (2002) original analyses of the less-is-more effect for the case of imperfect memory, Katsikopoulos (2010) showed that for the less-is-more effect to occur the recognition validity does not need to be larger than the knowledge validity.

\section{RELATED JUDGMENT PHENOMENA BASED ON MEMORY OF PREVIOUS ENCOUNTERS}

In this final section, we connect the recognition heuristic to several classical phenomena (such as the reiteration effect and the mere exposure effect) in judgment research that also describe how memory of past exposure to objects can be exploited to make inferences about unknown aspects of the environment. What is different 
about the recognition heuristic, however, is its precise account of the process involved in making an inference. The purpose of this connection is to highlight how research on the recognition heuristic could inspire novel questions in the study of these phenomena and to demonstrate that the special status of recognition may pervade decision making more generally.

\section{INFERENCES ABOUT THE TRUTH OF A STATEMENT}

A common inference problem in the real world is to judge whether a statement encountered is correct or false. What is the role of recognition, or more generally memory traces created by previous encounters with a statement, when making such inferences? Hasher et al. (1977) presented participants, over a period of 3 weeks, with statements that were either true or false (e.g., "the People's Republic of China was founded in 1947"). Most of the statements appeared only once, but some were presented repeatedly across the three sessions. Hasher et al. (1977) found that when participants subsequently indicated their confidence that a statement was true, they expressed an increasing confidence in the veracity of a statement the more frequently it was repeated. This reiteration effect (or frequency-validity effect) can be taken to indicate that participants used the strength of the memory traces of the statements as an indication of how likely the statement was to be true.

The reiteration effect is closely related to findings by Gilbert and colleagues, who presented their participants with a series of statements followed by information as to whether each statement was true or false (Gilbert et al., 1990, 1993; Gilbert, 1991; but see Hasson et al., 2005). When participants had an uncertain basis for assessing the statement's veracity (e.g., because they were distracted during processing; Gilbert et al., 1990), participants showed a stronger tendency to misclassify a previously seen false statement as true than to misclassify a true statement as false. In contrast, previously unseen statements were often classified as false. So even single previous encounters may be used by people to infer something about a statement, namely, that it is true. Although this default to believe a previously seen statement can be overturned, making such a switch appears to require additional cognitive resources: when under time pressure or cognitive load, participants tended to treat as true even statements they were previously informed were false (Gilbert et al., 1993). This parallels the finding for the recognition heuristic that under time pressure people tend to ascribe recognized objects a higher criterion value than unrecognized objects even when recognition is a poor cue (Pachur and Hertwig, 2006). Interestingly, Gilbert et al. (1990) also mentioned that the initial belief in the truth of statements that one encounters "may be economical and... adaptive" (p. 612), thus offering a potential link to the concept of ecological rationality. Finally, in parallel with McCloy et al.'s (2010) demonstration of framing effects in recognition-based inference (see above), Gilbert (1991) argued that "acceptance is psychologically prior" to rejection of the truth of a statement (p. 116).

\section{ESTIMATION}

The decisions considered so far involved categorical judgments about the environment, such as, which is larger: A or B? Is the statement $\mathrm{X}$ true or false? But often we have to make an absolute estimate regarding some aspect of an object and come up with a numerical value (e.g., the number of inhabitants of a city). Is information about whether one has heard of an object also used for estimation? Results by Brown (2002), who studied people's estimations of dates of events and country sizes, suggest that they $\mathrm{do}^{5}$. Specifically, participants estimated unrecognized events as occurring a rather long time ago and unfamiliar countries as having rather small populations. People thus seem to take their ignorance as useful information for where to locate an object on a quantitative dimension even in absolute estimation. Compared to the recognition heuristic, the processes involved in estimation are probably more complex, using metric and distribution knowledge to convert ignorance into a quantitative estimate. Lee and Brown (2004) proposed a model describing how people make date estimates of unknown events by combining the fact that they are not recognized with other information provided by the task.

\section{PREFERENCE AND ASCRIPTION OF POSITIVE MEANING}

So far we have looked at recognition-based inferences about objective characteristics of the environment. What about the effects of previous encounters on preferences, for which there is no objective criterion? As shown in the mere exposure effect (Zajonc, 1968), repeatedly encountering an object results in an increased liking or preference for the object. In addition, objects such as symbols are generally ascribed a more positive meaning the more often they have been encountered. This indicates that memory traces of previous encounters are also used for constructing one's affective responses to the environment. However, it is important to stress that in contrast to the recognition heuristic, these effects do not require that the object is recognized as having been seen before. Hence, the recognition heuristic cannot account for the mere exposure effect. The fluency heuristic (Schooler and Hertwig, 2005) is one possible mechanism by which (consciously) unrecognized objects may gain preference through repeated exposure (and the same process may also apply to inferences between unrecognized objects).

In a direct test of the recognition heuristic in preference judgments, Oeusoonthornwattana and Shanks (2010) first taught participants information about various brands (e.g., "Ecover has been engaging in animal testing.") and then asked them whether they would rather pick a product (e.g., shower gel) of a recognized brand - for which they had learned additional knowledge - or a product of an unrecognized brand. The authors concluded that recognition had a large influence on the preferences, but a large majority of participants also considered the taught knowledge. In fact, however, it is rather unclear what these findings mean for the recognition heuristic. As in most studies on the recognition heuristic, Oeusoonthornwattana and Shanks (2010) tested no alterative model. So although participants sometimes did not rely on the recognition heuristic, it may still provide the best available model for the observed data. In addition, the taught knowledge about the brands concerned information about the ethical standards of the brands, which might receive special attention compared to more common product information. Due to these methodological problems, we still know relatively little about the role of the recognition heuristic in consumer preferences. In fact, several authors have

${ }^{5}$ A similar observation was made by Pachur and Hertwig (2006): in an estimation task, people assigned unrecognized diseases to intermediate, rather than extremely low, frequency categories. 
emphasized the apparently dominant impact of brand recognition in consumer choice (e.g., Allison and Uhl, 1964; Hoyer and Brown, 1990).

\section{CONCLUSION}

The recognition heuristic was proposed as a model of how recognition, reflecting a particular statistical structure related to experiencing objects in the environment, can be exploited by a smart and simple mechanism to make inferences about those

\section{REFERENCES}

Allison, R. I., and Uhl, K. P. (1964). Influence of beer brand identification on taste perception. J. Mark. Res. $1,36-39$.

Anderson, J. R. (1974). Retrieval of propositional information from long-term memory. Cogn. Psychol. 5, 451-474.

Anderson, J. R., Bothell, D., Byrne, M. D., Douglass, S., Lebiere, C., and Qin, Y. (2004). An integrated theory of the mind. Psychol. Rev. 111, 1036-1060.

Ayton, P., and Önkal, D. (2004). Effects of Ignorance and Information on Judgmental Forecasting. London: City University (unpublished manuscript).

Borges, B., Goldstein, D. G., Ortmann, A., and Gigerenzer, G. (1999). "Can ignorance beat the stock market?" in Simple Heuristics That Make us Smart, eds G. Gigerenzer, P. M. Todd, and The ABC Research Group (New York: Oxford University Press), 59-72.

Boyd, M. (2001). On ignorance, intuition, and investing: a bear market test of the recognition heuristic. J. Psychol. Financ. Markets 2, 150-156.

Bröder, A., and Eichler, A. (2006). The use of recognition information and additional cues in inferences from memory. Acta Psychol. (Amst.) 121, 275-284.

Brown, N. R. (2002). Real-world estimation: estimation modes and seeding effects. Psychol. Learn. Motiv. 41, 321-359.

Brunswik, E. (1943). Organismic achievement and environmental probability. Psychol. Rev. 50, 255-272.

Bullock, S., and Todd, P. M. (1999). Made to measure: ecological rationality in structured environments. Minds Mach. 9, 497-541.

Davis-Stober, C. P., Dana, J., and Budescu, D. V. (2010). Why recognition is rational: optimality results on singlevariable decision rules. Judgm. Decis. Mak. 5, 216-229.

Dougherty, M. R., Franco-Watkins, A. M., and Thomas, R. (2008). Psychological plausibility of the theory of probabilistic mental models and the fast and frugal heuristics. Psychol. Rev. 115, 199-213.
Erdfelder, E., Küpper-Tetzel, C. E., and Mattern, S. D. (2011). Threshold models of recognition and the recognition heuristic. Judgm. Decis. Mak. 6, 7-22.

Ewald, P.W.(1994). Evolution of Infectious Diseases. Oxford: Oxford University Press.

Ford, J. K., Schmitt, N., Schechtman, S. L., Hults, B. H., and Doherty, M. L. (1989). Process tracing methods: contributions, problems, and neglected research questions. Organ. Behav. Decis. Process. 43, 75-117.

Frosch, C., Beaman, C. P., and McCloy, R. (2007). A little learning is a dangerous thing: an experimental demonstration of ignorance-driven inference. Q. J. Exp. Psychol. 60, 1329-1336.

Galef, B. G. Jr., McQuoid, L. M., and Whiskin, E. E. (1990). Further evidence that Norway rats do not socially transmit learned aversions to toxic baits. Anim. Learn. Behav. 18, 199-205.

Gigerenzer, G., and Brighton, H. (2009). Homo heuristicus: why biased minds make better inferences. Top. Cogn. Sci. 1, 107-143.

Gigerenzer, G., and Goldstein, D. G. (1996). Reasoning the fast and frugal way: models of bounded rationality. Psychol. Rev. 103, 650-669.

Gigerenzer, G., and Goldstein, D. G. (2011). The recognition heuristic: a decade of research. Judgm. Decis. Mak. 6, 100-121.

Gigerenzer, G., Hoffrage, U., and Kleinbölting, H. (1991). Probabilistic mental models: a Brunswikian theory of confidence. Psychol. Rev. 98, 506-528.

Gigerenzer, G., Todd, P. M., and The ABC Research Group. (1999). Simple Heuristics That Make us Smart. New York: Oxford University Press.

Gilbert, D. T. (1991). How mental systems believe. Am. Psychol. 46, 107-119.

Gilbert, D. T., Krull, D. S., and Malone, P. S. (1990). Unbelieving the unbelievable: some problems in the rejection of false information. J. Pers. Soc. Psychol. 59, 601-613.

Gilbert,D. T., Tafarodi, R. W., and Malone, P. S. (1993). You can't not believe everything you read. J. Pers. Soc. Psychol. $65,221-233$

objects. By virtue of its precise formulation allowing for clear-cut predictions, the recognition heuristic has been the focus of a large number of studies in a relatively short time. The studies indicate that a majority of people consistently rely on the recognition heuristic when it is ecologically rational, signaling its adaptive use. It thus offers perhaps the simplest realization of Herbert Simon's notion that boundedly rational decision making can arise from simple mental tools that are matched to the structure of the environment.

Goldstein, D. G., and Gigerenzer, G. (1999). "The recognition heuristic: how ignorance makes us smart," in Simple Heuristics That Make us Smart, eds G. Gigerenzer, P. M. Todd, and The ABC Research Group (New York: Oxford University Press), 37-58.

Goldstein, D. G., and Gigerenzer, G. (2002). Models of ecological rationality: the recognition heuristic. Psychol. Rev. 109, 75-90.

Goldstein,W.M., and Weber, E. U. (1997) "Content and discontent: indications and implications of domain specificity in preferential decision making," in Research on Judgment and Decision Making: Currents, Connections and Controversies, eds W. M. Goldstein and R. M. Hogarth (Cambridge: Cambridge University Press), 566-617.

Hasher, L., Goldstein, D., and Toppino, T. (1977). Frequency and the conference of referential validity. J. Verbal Learn. Verbal Behav. 16, 107-112.

Hasson, U., Simmons, J. P., and Todorov, A. (2005). Believe it or not: on the possibility of suspending belief. Psychol. Sci. 16, 566-571.

Hertwig, R., Herzog, S. M., Schooler, L. J., and Reimer, T. (2008). Fluency heuristic: a model of how the mind exploits a by-product of information retrieval. J. Exp. Psychol. Learn. Mem. Cogn. 34 1191-1206.

Hertwig, R., Pachur, T., and Kurzenhäuser, S. (2005). Judgments of risk frequencies: tests of possible cognitive mechanisms. J. Exp. Psychol. Learn. Mem. Cogn. 31, 621-642.

Hertwig, R., and Todd, P. M. (2003) "More is not always better: the benefits of cognitive limits," in Thinking: Psychological Perspectives on Reasoning, Judgment and Decision Making, eds D. Hardman and L. Macchi (Chichester: Wiley), 213-231.

Hilbig, B. E., Erdfelder, E., and Pohl, R. F. (2010a). One-reason decisionmaking unveiled: a measurement model of the recognition heuristic. J. Exp. Psychol. Learn. Mem. Cogn. 36, 123-134.

Hilbig, B. E., Scholl, S. G., and Pohl, R. (2010b). Think or blink: is the recogni- tion heuristic an "intuitive" strategy? Judgm. Decis. Mak. 5, 272-284.

Hilbig, B. E., and Pohl, R. F. (2008). Recognizing users of the recognition heuristic. Exp. Psychol. 55, 394-401.

Hilbig, B. E., and Pohl, R. F. (2009). Ignorance-versus evidence-based decision making: a decision time analysis of the recognition heuristic. J. Exp. Psychol. Learn. Mem. Cogn. 35, 1296-1305.

Hilbig, B. E., Pohl, R. F., and Bröder, A. (2009). Criterion knowledge: a moderator of using the recognition heuristic? J. Behav. Decis. Mak. 22, 510-522.

Hintzman, D. L., and Curran, T. (1994). Retrieval dynamics of recognition and frequency judgments: evidence for separate processes of familiarity and recall. J. Mem. Lang. 33, 1-18.

Hoyer, W. D., and Brown, S. P. (1990). Effects of brand awareness on choice for a common, repeat-purchase product. J. Consum. Res. 17, 141-148.

Jacoby, L. L., and Dallas, M. (1981). On the relationship between autobiographical memory and perceptual learning. J. Exp. Psychol. Gen. 110, 306-340.

Jacoby, L. L., Kelley, C., Brown, J., and Jasechko, J. (1989). Becoming famous overnight: limits on the ability to avoid unconscious influences of the past. $J$. Pers. Soc. Psychol. 56, 326-338.

Johnson, M. K., Hastroudi, S., and Lindsay, D. S. (1993). Source monitoring. Psychol. Bull. 114, 3-28.

Katsikopoulos, K. V. (2010). The lessis-more effect: predictions and tests. Judgm. Decis. Mak. 5, 244-257.

Kelley, C. M., and Jacoby, L. L. (1998). Subjective reports and process dissociation: fluency, knowing and feeling. Acta Psychol. (Amst.) 98, 127-140.

Lee, P. J., and Brown, N. R. (2004). The role of guessing and boundaries on date estimation biases. Psychon. Bull. Rev. 11, 748-754.

Marewski, J. N., Gaissmaier, W., Dieckmann, A., Schooler, L. J., and Gigerenzer, G. (2005). Ignorancebased reasoning? Applying the recognition heuristic to elections. Paper Presented at the 20th Biennial Conference on Subjective Probability, 
Utility and Decision Making, Stockholm.

Marewski, J. N., Gaissmaier, W., Schooler, L. J., Goldstein, D. G., and Gigerenzer, G. (2010). From recognition to decisions: extending and testing recognition-based models for multialternative inference. Psychon. Bull. Rev. 17, 287-309.

Marewski, J. N., and Schooler, L. J. (in press). Cognitive niches: an ecological model of strategy selection. Psychol. Rev.

Mata, R., Schooler, L. J., and Rieskamp, J. (2007). The aging decision maker: cognitive aging and the adaptive selection of decision strategies. Psychol. Aging 22, 796-810.

McCloy, R., Beaman, C. P., Frosch, C. A., and Goddard, K. (2010). Fast and frugal framing effects? J. Exp. Psychol. Learn. Mem. Cogn. 36, 1043-1052.

Newell, B. R., and Fernandez, D. (2006). On the binary quality of recognition and the inconsequentiality of further knowledge: two critical tests of the recognition heuristic. J. Behav. Decis. Mak. 19, 333-346.

Newell, B. R., and Shanks, D. R. (2004). On the role of recognition in decision making. J. Exp. Psychol. Learn. Mem. Cogn. 30, 923-935.

Noble, J., Todd, P. M., and Tuci, E. (2001). Explaining social learning of food preferences without aversions: an evolutionary simulation model of Norway rats. Proc. R. Soc. Lond. B Biol. Sci. 268, 141-149.

Oeusoonthornwattana, O., and Shanks, D. R. (2010). I like what I know: is recognition a non-compensatory determiner of consumer choice? Judgm. Decis. Mak. 5, 310-325.

Oppenheimer, D. M. (2003). Not so fast! (and not so frugal!): rethinking the recognition heuristic. Cognition 90, B1-B9.

Oppenheimer,D.M. (2004). Spontaneous discounting of availability in fre- quency judgment tasks. Psychol. Sci. $15,100-105$.

Oppenheimer, D. M. (2008). The secret life of fluency. Trends Cogn. Sci. (Regul. Ed.) 12, 237-241.

Ortmann, A., Gigerenzer, G., Borges, B., and Goldstein, D. G. (2008). "The recognition heuristic: a fast and frugal way to investment choice?" in Handbook of Experimental Economics Results: Vol. 1 (Handbooks in Economics No. 28), eds C. R. Plott and V. L. Smith (Amsterdam: NorthHolland), 993-1003.

Pachur, T. (2010). Recognition-based inference: when is less more in the real world? Psychon. Bull. Rev. 17, 589-598.

Pachur, T. (in press). The limited value of precise tests of the recognition heuristic. Judgm. Decis. Mak.

Pachur, T., and Biele, G. (2007). Forecasting from ignorance: the use and usefulness of recognition in lay predictions of sports events. Acta Psychol. (Amst.) 125, 99-116.

Pachur, T., Bröder, A., and Marewski, J. N. (2008). The recognition heuristic in memory-based inference: is recognition a non-compensatory cue? J. Behav. Decis. Mak. 21, 183-210.

Pachur, T., and Hertwig, R. (2006). On the psychology of the recognition heuristic: retrieval primacy as a key determinant of its use. $J$. Exp. Psychol. Learn. Mem. Cogn. 32, 983-1002.

Pachur, T., Mata, R., and Schooler, L. J. (2009). Cognitive aging and the use of recognition in decision making. Psychol. Aging 24, 901-915.

Pleskac, T. J. (2007). A signal detection analysis of the recognition heuristic. Psychon. Bull. Rev. 14, 379-391.

Pohl, R. (2006). Empirical tests or the recognition heuristic. J. Behav. Decis. Mak. 19, 251-271.

Reimer, T., and Katsikopoulos, K. V. (2004). The use of recognition in group decision-making. Cogn. Sci. 28, 1009-1029.

Richter, T., and Späth, P. (2006). Recognition is used as one cue among others in judgment and decision making. J. Exp. Psychol. Learn. Mem. Cogn. 32, 150-162.

Scheibehenne, B., and Bröder, A. (2007). Predicting Wimbledon 2005 tennis results by mere player name recognition. Int. J. Forecast. 23, 415-426.

Schooler, L., and Hertwig, R. (2005). How forgetting aids heuristic inference. Psychol. Rev. 112, 610-628.

Schwarz, N., Bless, H., Strack, F., Klumpp, G., Rittenauer-Schatka, H., and Simons, A. (1991). Ease of retrieval as information: another look at the availability heuristic. J. Pers. Soc. Psychol. 61, 195-202.

Schwarz, N., and Vaughn, L. A. (2002). "The availability heuristic revisited: ease of recall and content of recall as distinct sources of information," in Heuristics and Biases: The Psychology of Intuitive Judgment, eds T. Gilovich, D. Griffin, and D. Kahneman (New York: Cambridge University Press), 103-119.

Serwe, S., and Frings, C. (2006). Who will win Wimbledon 2003? The recognition heuristic in predicting sports events. J. Behav. Decis. Mak. 19, 321-332.

Simon, H.A. (1990). Invariants of human behavior. Annu. Rev. Psychol.41, 1-19.

Smithson, M. (2010). When less is more in the recognition heuristic. Judgm. Decis. Mak. 5, 230-243.

Snook, B., and Cullen, R. M. (2006). Recognizing national hockey league greatness with an ignorance-based heuristic. Can. J. Exp. Psychol. 60, 33-43.

Todd, P. M., and Heuvelink, A. (2007). "Shaping social environments with simple recognition heuristics," The Innate Mind, Vol. 2, Culture and Cognition, eds P. Carruthers,
S. Laurence, and S. Stich (Oxford: Oxford University Press), 165-180.

Tversky, A., and Kahneman, D. (1973). Availability: a heuristic for judging frequency and probability. Cogn. Psychol. $5,207-232$.

Volz, K. G., Schooler, L. J., Schubotz, R. I., Raab, M., Gigerenzer, G., and von Cramon, D. Y. (2006). Why you think Milan is larger than Modena: neural correlates of the recognition heuristic. J. Cogn. Neurosci. 18, 1924-1936.

Weber, E. U., Siebenmorgen, N., and Weber, M. (2005). Communicating asset risk: how name recognition and the format of historic volatility information affect risk perception and investment decisions. Risk Anal. 25, 597-609.

Zajonc, R. B. (1968). Attitudinal effects of mere exposure. J. Pers. Soc. Psychol. 9, 1-27.

Conflict of Interest Statement: The authors declare that the research was conducted in the absence of any commercial or financial relationships that could be construed as a potential conflict of interest.

Received: 27 January 2011; paper pending published: 23 April 2011; accepted: 17 June 2011; published online: 05 July 2011.

Citation: Pachur T, Todd PM, Gigerenzer G, Schooler LJ and Goldstein DG (2011) The recognition heuristic: a review of theory and tests. Front. Psychology 2:147. doi: 10.3389/ fpsyg.2011.00147

This article was submitted to Frontiers in Cognitive Science, a specialty of Frontiers in Psychology.

Copyright $\odot 2011$ Pachur, Todd, Gigerenzer, Schooler and Goldstein. Thisisanopen-access article subject to a non-exclusive license between the authors and Frontiers Media $S A$, which permits use, distribution and reproduction in other forums, provided the original authors and source are credited and other Frontiers conditions are complied with. 